\title{
Deep compositional robotic planners that follow natural language commands
}

\author{
Yen-Ling Kuo, Boris Katz, and Andrei Barbu
}

\begin{abstract}
We demonstrate how a sampling-based robotic planner can be augmented to learn to understand a sequence of natural language commands in a continuous configuration space to move and manipulate objects. Our approach combines a deep network structured according to the parse of a complex command that includes objects, verbs, spatial relations, and attributes, with a sampling-based planner, RRT. A recurrent hierarchical deep network controls how the planner explores the environment, determines when a planned path is likely to achieve a goal, and estimates the confidence of each move to trade off exploitation and exploration between the network and the planner. Planners are designed to have near-optimal behavior when information about the task is missing, while networks learn to exploit observations which are available from the environment, making the two naturally complementary. Combining the two enables generalization to new maps, new kinds of obstacles, and more complex sentences that do not occur in the training set. Little data is required to train the model despite it jointly acquiring a $\mathrm{CNN}$ that extracts features from the environment as it learns the meanings of words. The model provides a level of interpretability through the use of attention maps allowing users to see its reasoning steps despite being an end-to-end model. This end-to-end model allows robots to learn to follow natural language commands in challenging continuous environments.
\end{abstract}

\section{INTRODUCTION}

When you carry out a command uttered in natural language, you combine your knowledge about the task to be performed and how it was carried out in the past with reasoning about the consequences of your actions. Thinking about the task allows you to choose actions that are likely to make progress, and it is most useful when the path forward is clearly understood in an environment that has been experienced before. Thinking about the consequences of your actions allows you to handle new environments and obstacles, and it is most useful where a task must be performed in a novel way. Generally, prior work has excelled at one of these but not both. Powerful models can control agents but do so from moment to moment without planning complex actions [1, 2, 3]. Planners on the other hand efficiently explore configuration spaces, often by building search trees [4, 5], but require a target final configuration [6] [7, 8] or a symbolic specification of constraints [9, 10].

We demonstrate an end-to-end model that both reasons about a task and plans its action in a continuous domain resulting in a robot that can follow linguistic commands ${ }^{1}$

This work was supported by the Center for Brains, Minds and Machines, NSF STC award 1231216, the Toyota Research Institute, the DARPA GAILA program, the ONR Science of Artificial Intelligence, and CBMM-Siemens Graduate Fellowship.

CSAIL and CBMM, MIT \{ylkuo, boris, abarbu\}@mit.edu

${ }^{1}$ We will release the source code and models upon publication.

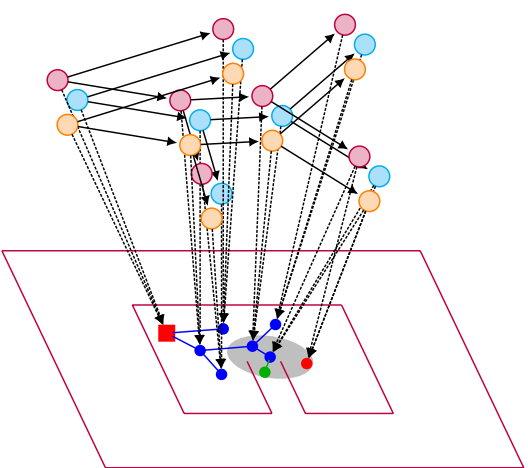

Fig. 1. We augment a sampling-based planner, RRT, with a hierarchical recurrent network that encodes the meaning of a natural-language command the robot must follow. Just as with a traditional planner, the robot mentally explores the space around a start location building a search tree to find a good path in its configuration space. Unlike a traditional planner, we do not specify a goal as a location, but instead rely on a neural network to score how likely any position in the configuration space is to be an end state while considering the past history of the robot's actions and its observation of the environment. The structure of the RNNs mirrors that of the search tree, with each splitting off as different decisions are considered. At each time step, the RNNs observe the environment, and can adjust the sampling process of the planner to avoid moving in undesirable locations (in this case, the tree is not expanded toward the red circle, and instead adjusted to go down the passageway through the green circle). See fig. 2 for details on the structured RNNs and how they encode the structure of sentences as relationships between recurrent models.

This is the first model to perform end-to-end navigation and manipulation tasks given natural language commands in continuous environments without symbolic representations. It integrates a planner with a compositional hierarchical recurrent network. The recurrent network learns which actions are useful toward a goal specified in natural language while the planner provides resilience when the situation becomes unclear, novel or too complicated. This process frees the network from having to learn the minutia of planning and allows it to focus on the overall goal while gaining robustness to novel environments.

To execute a command, the model proceeds as a traditional sampling-based planner with an additional input of a natural language command; see fig. 11. A collection of networks are arranged in a hierarchy that mirrors the parse of the command. This encodes the command into the structure of the model. A search tree is created through a modified RRT [11] which explores different configurations of the robot and their effect on the environment. The search procedure is augmented by the hierarchical network which can influence the nodes being expanded and the direction of expansion. As the search tree splits into multiple branches, the hierarchical recurrent network similarly splits following the tree. This encodes 
the reasoning and state of the robot if it were to follow that specific set of actions. At each time point, the network predicts the likelihood that the action satisfies the command. In the end, much as with a classical sampling-based planner, a search tree is created that explores different options, and a path in that tree is selected to be executed.

Robustness to new environments is achieved by trading off the planner against the hierarchical network. The influence of the network is proportional to its confidence. When new obstacles, map features, or other difficulties are encountered (for example, not immediately seeing a goal), the algorithm can temporarily devolve into a traditional RRT. This is a desirable feature because algorithms like RRT make optimal decisions when other guidance is not available. Unlike planners, uncertain or untrained networks generally make pathologically bad decisions in such settings. This issue is often alleviated with techniques such as $\epsilon$-greedy learning [12] which provide arbitrary random moves rather than the nearoptimal exploration that sampling-based planners engage in.

We ensure that the model provides a level of interpretability in two ways. First, the structure of the sentence is encoded explicitly into the structure of the recurrent network; see fig. 2 . Inspecting the network reveals which subnetworks are connected together and the topology of the connections mirrors that of natural language. Second, the internal reasoning of the model is highly constrained to operate through attention maps. Rather than allowing each component the freedom to pass along any information up the hierarchy in order to make a decision, we constrain all components to communicating via a grayscale map that is multiplied by the current observation of the environment. Inspecting these attention maps reveals information about which areas each network is focused on and can provide a means to understand and explain failures. In addition, this constrained representation is easy to learn and does not require a large number of examples. We find that adding these interpretable computations also increases performance relative to more opaque representations, likely because words which have never co-occurred at training time have an easier time understanding the output of other word models when the representations are interpretable.

This work makes four contributions.

1) We demonstrate how a robotic planner, in addition to reasoning about physical affordances and obstacles, can be extended to reason about a linguistic command in a continuous environment.

2) We show that a hierarchical recurrent model structured according to the parse of a sentence can learn the meanings of sentences efficiently thereby guiding a robot's motion and manipulation.

3) We demonstrate that such a model generalizes to new settings, to more challenging maps that include obstacles not seen in the training set, and to longer commands.

4) By constraining the model to reason visually through attention maps rather than arbitrary vectors, we produce an end-to-end model with more interpretable intermediate reasoning steps without needing intermediate symbolic representations.

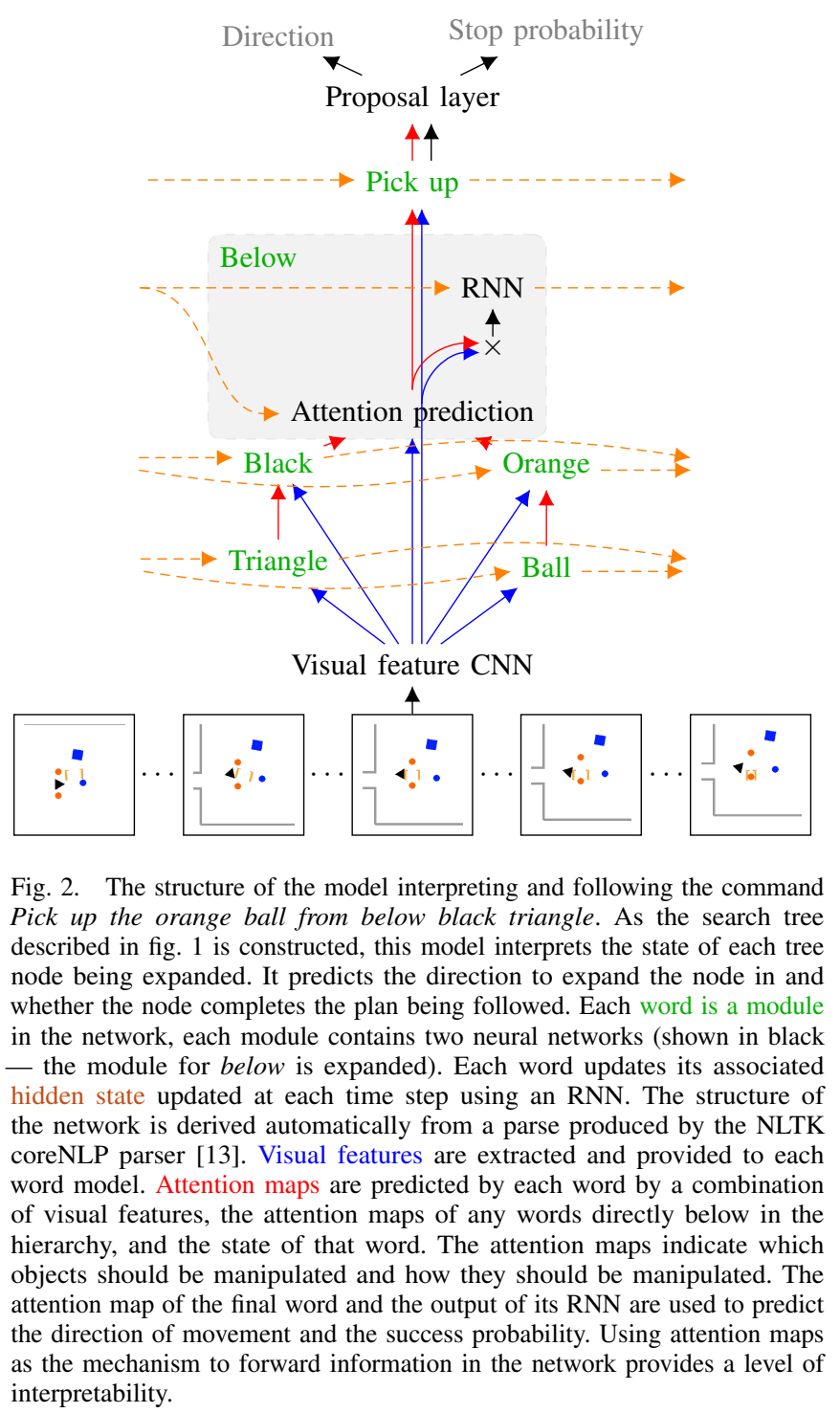

\section{RELATED WORK}

Much prior work has explored how symbolic representations in high-level planning languages, such as PDDL [14], can ground linguistic commands. In addition some approaches combine together task planning and motion planning using symbolic representations for the task [15, 16, 17, 18]. Such approaches can plan efficiently in large continuous spaces but require a symbolic representation of a task, must be manually created, cannot be trained to acquire new concepts, and do not handle ambiguity well. Our approach similarly combines task and motion planning, but does so without symbolic representations albeit with simpler tasks than stateof-the-art models in such domains can handle. Prior work has built models that can robustly follow linguistic commands on top of these architectures [9, 10]. Because of the underlying symbolic nature of the final representation, this prior work cannot acquire concepts that are not easily expressed in the target planning language and cannot learn new primitives in that language. Paxton et al. [19] demonstrate a model which breaks down tasks for such planners automatically - it learns 
to map sentences to a sequence of subgoals. Bisk et al. [20] demonstrate how to break down manipulation tasks and how to ground them to perception from natural language input, much like the tasks we perform here, but do not execute such commands.

Fu et al. [21] and Shah et al. [3] demonstrate an approach which can map sentences to robotic actions (navigation \& pick and place) via multi-task reinforcement learning in a discrete state and action space. This is similar in spirit to our approach but we do so in continuous action and state spaces which require many precise steps in the configuration space to execute what would otherwise be a single output token such as "pick up" for discrete problems.

Blukis et al. [1] demonstrate how a drone can be controlled by predicting the goal configuration of the robot. Their model operates in a continuous space but does not contain object interactions, manipulations, or obstacles. Predicting a single final goal for such complex multistep actions is infeasible, as the goal must contain not just the position of the robot but the position of the other objects.

\section{DEEP PLANNERS WITH LANGUAGE}

We describe how an extension of RRT adds neural networks that control the search process, section III-A, then describe the structure of the model used and how it encodes complex sentences, section III-B, and finally how such networks can be efficiently trained, section III-C.

\section{A. Planning with Deep RRT}

Robotic planners are efficient at searching the configuration spaces of robots. Here we describe how to augment them with networks that efficiently learn language, how to guide the planning process, and how to recognize when a plan described by a sentence has been completed. This is related to the approach of Kuo et al. [7], DeRRT, which introduced deep sequential models for sampling-based planning. It took an RRT-based planner and described how to guide its behavior with a neural network.

The planner maintains a search tree and a corresponding recurrent neural network with the goal of reaching a fixed destination in the configuration space. This is related to the model shown in fig. 1 - in this work, we use a collection of networks and have the networks determine the final configuration based on the command rather than explicitly providing it. The goal of a traditional planner is to construct a search tree that explores the space and connects the start state to an end state. It will then choose the best path between the two from the tree for the robot to follow.

At each step, the planner chooses a node to extend by the standard RRT mechanism: sampling a point in space and finding the nearest point in the tree to that sample. It then proposes a new tree node between the selected tree node and the sampled point. The neural network takes as input the state at the current node, any visual observations at the current node, and the proposed extension to the tree. Observations are processed with a co-trained CNN that is shared among all words. The network makes its own prediction about how to extend the tree at the current node along with a confidence value. A simple mixture model selects between the planner and the network proposed directions. Once the tree is constructed, in this case after a fixed number of planning steps, the node which is considered most likely to be an end state of the described command is chosen and the path between the start state and that node is generated.

We modify this algorithm to influence the choice of which node to expand, not just the direction to expand a preselected node in. These modifications can be applied to the original version of the algorithm presented in that earlier work. The neural network that guides the planner is trained to maximize the likelihood of the data provided. This results in a probability assigned to each node and each path. Every search tree node is annotated with its likelihood conditioned on its parent as well was with the likelihood of being chosen for expansion by the vanilla RRT. The latter probability is computed by generating many free-space samples and computing the distribution over which nodes are extended this is very computationally efficient. To then sample which node to extend, we multiply and normalize these probabilities, sampling from the distribution of nodes which would be chosen by both the network and RRT. This focuses search in areas where plans are likely to succeed while not allowing the neural network to get stuck in one region - as a region is more saturated with samples the likelihood that RRT would continue to extend the tree in it becomes very low.

\section{B. Language and Deep RRT}

The model described thus far uses a single recurrent network in order to guide the planner. Technically, this is serviceable, as the network can in principle learn to perform this task. Practically, generalizing to new sentences and complex sentential structures is beyond the abilities of that simple model.

Using a collection of networks, rather than a single network can help generalization to new sentences. Just as one network can guide a planner, multiple networks can also guide it. Each network can make a prediction. A direction can be sampled from the posterior distribution over all of the predictions by all of the networks.

We build this collection of networks out of a lexicon of component networks. Given a training set of commands and paths, one component network is trained per word in the command that the robot is following. Given a test sentence, the words in the sentence determine the set of component networks which guide the planner. We call this the bag of words, BoW, model because there is no explicit relationship or information flow between the networks. Due to the lack of relationships between words, this model has fundamental difficulties representing the difference between Grab the black toy from the box and Grab the toy from the black box.

To address this limitation, we introduce a hierarchical network; see fig. 2. Given a sentence and the parse of the sentence derived from the NLTK coreNLP parser [13], we select the same set of component networks that correspond to the words in the sentence. Networks are arranged in a tree 
- naturally, such trees are rooted by verbs in most linguistic representations. The state at the current node informs the representation of each component network. Each component updates its own hidden state and forwards information to all of the components that are linked to it. The leaves only receive as input observation at the current state and their own hidden state. The root of the tree produces an output used by a linear proposal layer to predict the direction of movement and the likelihood that the current node has reached a goal. This approach has the ability to represent the earlier distinction about which noun the word black is attached to because different attachments result in different parse trees and thus different instantiations of the model. One must pay particular attention to argument structure here - verbs which take multiple arguments such as give must always take them in the same order (the object of the given and the destination of the give should always fill the slots of give).

We restrict nodes to communicating via attention maps rather than arbitrary vectors. This helps generalization as words which never co-occurred in the training set can be seen in the test set of the experiments we report. By ensuring that the representation shared between component networks is universal, such as the attention maps, component networks are encouraged to be more compatible with one another. This is enforced by the structure of each component network, i.e., each network corresponding to a word. Each word takes as input a set of attention maps, weighs the input image with each attention map independently, and combines this with the hidden state of that word. A new attention map is predicted and passed to subsequent words. Using this predicted attention map, an RNN takes as input the observed image weighted by the attention map and updates the hidden state of the word. In addition to encouraging generalization, attention maps can be interpreted by humans, and help speed up learning by being relatively low dimensional.

\section{Training Compositional Deep RRT}

There are three parts of the model that must be trained: the shared CNN that embeds visual observations, a lexicon of component networks, and the proposal layer. The lexicon of component networks maps words to networks that represent the meanings of those words. We might in principle annotate when a word is relevant to a plan and train each word independently, but we find that joint training allows easier and already-known words to supervise new words. This is because the hierarchical nature of the model allows information flow between words, giving words which have high confidence an opportunity to help guide the representation of words which are not yet well trained. Instead, we train the model with little supervision: pairs of sentences and paths. The model is not informed about which parts of the sentence correspond to which parts of the path, when words are relevant, or how words relate to visual observations.

The overall model is trained in two phases. First, all weights are trained including the shared $\mathrm{CNN}$ that embeds visual observations, the lexicon of component networks, and the direction in which to extend the search tree. Next, these three

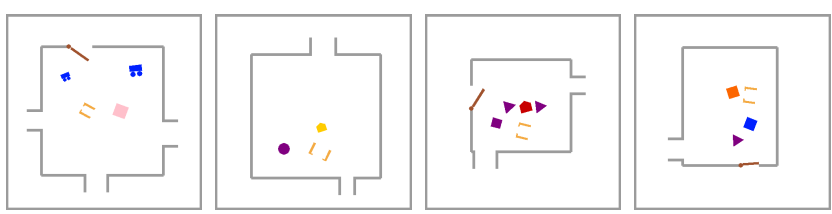

(a) Representative examples of the training set

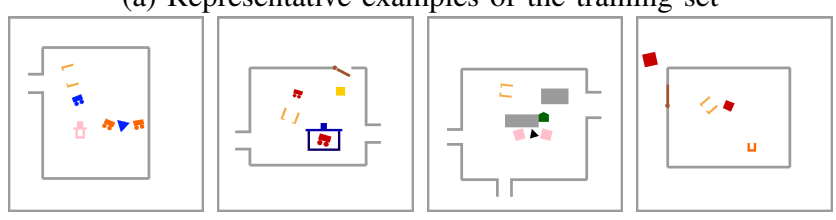

(b) Representative examples of the test set

Fig. 3. Examples of the (a) training set and of the (b) test set. The robot is shown in orange as a pair of L-shaped grippers. Objects are randomly positioned, with random properties and orientations. The training set is considerably simpler, with fewer objects on average, without cups that have lids, without the need to traverse doors or channels as all objects are inside the room, and without immovable obstacles (grey rectangles).

sets of weights are fixed while the proposal layer is fine-tuned to predict the likelihood of a state being a goal state of a plan. This fine-tuning step significantly increase performance without requiring more training data: the proposal layer gains experience with how to interpret the output of the network without the network also changing its behavior.

The model presented here and summarized in fig. 1 and fig. 2 is trained with little data, only sentences paired with demonstrations. It operates efficiently in continuous environments. Its structure is intelligible and derived from linguistic principles while its reasoning is made overt by the explicit use of attention maps. Next, we describe how we evaluate this model.

\section{EXPERIMENTS}

To evaluate the model, we describe the task and training set generation procedure, section IV-A then describe baseline models, section IV-B, test the ability of the model to carry out novel commands, section IV-C the ability to generalize to novel features in the environment, section IV-D, generalize to multiple sentences, section IV-E and the ability to handle real-world commands generated by users, section IV-F

\section{A. Dataset}

A generative model creates new training and test maps conditioned on a target command which is sampled from a grammar. The space of possible maps is large and includes rooms of varying sizes, which can have between 0 and 4 narrow gaps, possibly contain a door to the outside, and may contain between two and eight objects with multiple properties (shape, color, and size); see fig. 3. The grammar which generates commands contains seven verbs (push, grab, approach, touch, open, leave, carry), seven nouns (block, cup, ball, triangle, quadrilateral, house, cart), eight colors (red, green, blue, pink, yellow, black, purple, orange), two sizes (big, small), nine spatial relations (left of, right of, top of, bottom of, on the left of, on the right of, near, above, below), and two prepositions (towards, away from). Each 


$\begin{array}{ccc}\text { Planner } & \text { Two concepts } & \text { Five or six concepts } \\ \text { RNN-Only } & 0.25 & 0.24 \\ \text { BoW } & 0.61 & 0.36 \\ \text { Ours } & \mathbf{0 . 7 2} & \mathbf{0 . 5 0} \\ \text { RRT+Oracle } & 0.64 & 0.49\end{array}$

Fig. 4. Success rate of executing natural language commands with two concepts, the same number as the models saw at training time, and five or six concepts, more complex sentences than were seen at training time. All models sampled 500 nodes in the configuration space of the robot. Our model generalizes well and faithfully encodes the meaning of commands. While the BoW model is also novel it lacks the internal structure to represent many sentences and significantly underperforms our hierarchical model.

of these linguistic constituents becomes a component neural network in a lexicon of networks. Sentences are parsed with the NLTK coreNLP parser [13] and unknown words in the sentences are mapped to nearby words using their distance in WordNet [22, 23].

Given all of the possible objects, distractors, room sizes, doors, gateways, object locations, color, rotation, and size, a random map is generated. We verify that the target plan is in principle feasible on this map. The same map never appears in both the training and the test sets. This provides an immense space from which to generate maps and to test model generalization capabilities.

\section{B. Models}

As described in the related work, section III no existing model is able to take as input linguistic commands and plan in the environments used here. To evaluate our model, we develop several baselines by augmenting earlier work to perform this challenging task.

The weakest baseline, $R N N$-Only, is our model without the planner but including the hierarchical neural network. A more powerful baseline, $B o W$, is created by augmenting the work in Kuo et al. [7]. The network described there is given the added task of predicting when a configuration of the robot is a terminal. A collection of neural networks represent the meaning of a sentence, but they do not interact with one another; these form the bag of words. This model is novel and related to our own, but considerably weaker as there is no relationship between the words and no explicit encoding of the structure of the sentence. The neural network used in that is also modified to predict both a direction to move in and the probability of ending the action - similarly to the model presented here but using a single neural network.

Finally, we compare against a model, $R R T+$ Oracle, which represents the performance that can be expected if the hierarchical network is operating well. This model uses the same underlying planner but the goal regions are manually specified through the use of an oracle. For any position in the configuration space of the robot and the configuration space of all of the objects, the oracle determines if the behavior of the robot has satisfied some natural language utterance. Equaling this strong related model in performance demonstrates that the network is acquiring the meanings of words.

\section{Understanding Natural Language Commands}

First, we test if our model can acquire the meanings of words and use this to represent never-before-seen sentences;

$\begin{array}{cccc}\text { Planner } & \text { Obstacles } & \text { Cup \& Lid } & \text { Door } \\ \text { RNN-Only } & 0.12 & 0.08 & 0 \\ \text { BoW } & 0.32 & 0.08 & \mathbf{0 . 3 5} \\ \text { Ours } & \mathbf{0 . 5 2} & \mathbf{0 . 1 6} & 0.30 \\ \text { RRT+Oracle } & \mathbf{0 . 5 2} & 0.08 & \mathbf{0 . 3 5}\end{array}$

Fig. 5. The success rate of different baselines and models when generalizing to environments that have properties which have never or rarely been experienced at training time. Note that, the RNN-Only model which does not include a planner, fails to generalize. Models which do include a planner generalize much better to new problems.

see fig. 4 Note that very little is annotated here: only pairs of demonstrations and sentences related to those demonstrations exist. Also note that for all experiments, test training and test maps and utterances were disjoint.

We generated a training set of 6099 utterances containing at most four concepts, with each utterance being demonstrated on a new map. The test set consisted of 657 utterances paired with maps that do not appear in the training set and are on the whole considerably more complex; see fig. 3 The model presented here had by far the highest success rate (72\%) and generalized best to more complex sentences. At training time, we never presented sentences that had more than four concepts, while at test time, we included considerably more complex sentences. Our model generalized to these longer sentences despite not having seen anything like them at training time.

Since our model affects the search direction of RRT, i.e., the growth of the search tree, it even outperformed the $R R T+$ Oracle model. The RRT+Oracle model has a perfect understanding of the sentence in terms of determining which nodes satisfy the command, but lacks the ability to use the sentence to guide its actions. This demonstrates that the model presented here faithfully encodes commands and executes them well in complex environments, on new maps, even when those commands are much more complex than those seen in the training set.

\section{Additional Obstacles and Preconditions}

Robots must continually deal with new difficulties. To evaluate the capacity of models to adapt to new problems, we further modify the test set to include other features not present at training time; see fig. 5. In particular, we add up to four random fixed obstacles and require that the robot traverse a push-button-controlled door. In addition, the frequency of objects inside cups with lids is significantly increased.

The model which does not include a planner, the RNN-Only model, has great difficulty generalizing to new scenarios. All the other models generalized far better, with ours performing roughly on par with the oracle. These results indicate that planners provide robustness when encountering new challenges. This is well known in symbolic planning but has not been exploited as part of an end-to-end approach before.

\section{E. Multiple Sentences}

Robots are unlikely to be required to carry out just one command at a time. Most plans will include a sequence of actions that depend on one another. We choose to evaluate 


\begin{tabular}{cccc} 
Planner & \multicolumn{3}{c}{ Number of sentences } \\
\cline { 2 - 4 } & 1 & 2 & 3 \\
RNN-Only & 0.17 & 0 & 0 \\
BoW & 0.40 & 0.12 & 0.06 \\
Ours & $\mathbf{0 . 5 8}$ & $\mathbf{0 . 3 3}$ & 0.10 \\
RRT+Oracle & 0.52 & 0.25 & $\mathbf{0 . 1 2}$
\end{tabular}

Fig. 6. All models are trained on a single utterance and are then required to follow a sequence of commands. Every model is allowed to sample 600 nodes in the configuration space of the robot. As more commands are added, carrying out a task becomes increasingly difficult and the RNN-Only model is quickly overwhelmed. The BoW model performs at roughly half of the performance of ours. Our model has performance comparable to that of the oracle. Sampling more nodes would increase the success rate of all models.

an extreme version of this task where all models are only trained on a single sentence and then must generalize to sequences of between two and three commands; see fig. 6 Despite this significant limitation at training time, we find that our approach outperforms the baselines significantly. The RNN-Only model is unable to generalize. The BoW model has roughly half the performance of our model. Our model has similar properties to that of the oracle, which has the correct encoding of the sentence, thus showing that our model represents sequences of sentences despite not being trained on any sequences of commands.

\section{F. User Study}

We generated 500 map and command pairs and had the robot execute those commands. The executions of these commands, but not the commands themselves, were shown to four users recruited for this experiment. Users were asked to produce the instructions they would provide to the robot in order to elicit the behavior they observed. Out of 500 descriptions, 128 were impossible for the robot to follow due to user error, e.g., by mentioning objects that are physically not there, or could not be reasonably parsed. The 372 remaining descriptions had an average length of 9.04 words per sentence standard deviation of 2.49. The baseline RNN-Only model achieved $17 \%$ success rate, the BoW model succeeded $40 \%$ of the time, while our model succeeded $49 \%$ of the time. The RRT+Oracle model had roughly the same performance as ours succeeding $51 \%$ of the time. This demonstrates that our approach scales to real-world user input.

\section{DISCUSSION \& CONCLUSION}

We have demonstrated that a hierarchical recurrent network can work in conjunction with a sampling-based planner to create a model that encodes the meaning of commands. It learns to execute novel commands in challenging new environments that contain features not seen in the training set. We demonstrated that our approach scales to real-world sentences produced by users.

Our model provides a level of interpretability. The structure of the model overtly mirrors that of the parse of a sentence making it easy to verify if a sentence has been incorrectly encoded. Attention maps are used throughout the hierarchical network to allow component parts to communicate with one another. These provide another means by which to

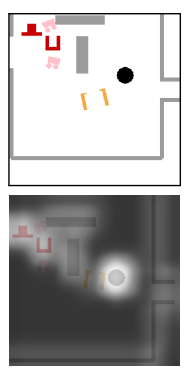

cup

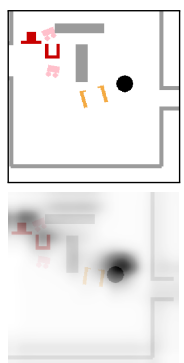

cart

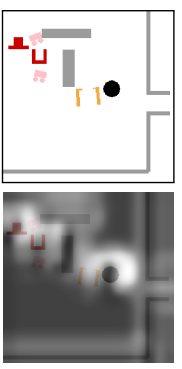

above

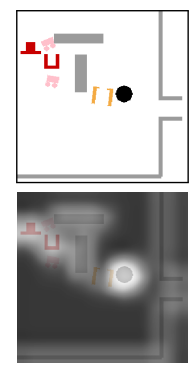

approach (a) Failure: Approach the cart above the cup

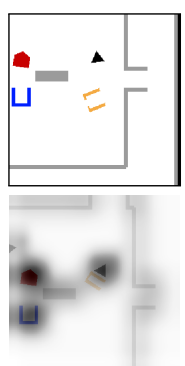

triangle

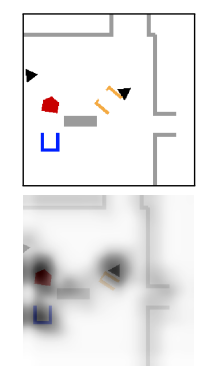

house

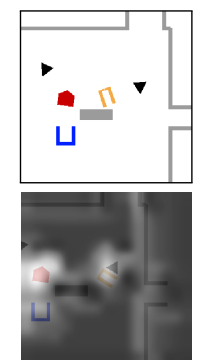

toward

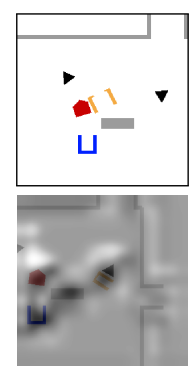

carry (b) Failure: Carry the triangle toward the house

Fig. 7. Examples of snapshots from the execution of the model (top; these are from the point of view of the robot and represent the local information available to it rather than the entire map) along with the attention maps (bottom) produced by each component network in the model at a critical time in the execution of two commands which were not carried out correctly. Note that the polarity of the attention maps is irrelevant - models can communicate by either suppressing or highlighting features and neither carries any a priori valence. In (a) the robot fails to pick up the correct object and heads to the circle instead. The failure in (a) is explained by the poor detection of the cup seemingly confusing it with the circle. In (b) the model goes to the triangle but then fails to pick it up before heading to the house. The failure is not explained by the attention maps, as they correctly highlight the relevant objects - instead the model seems to have positioned itself incorrectly to perform the pick up (which is attempted) and it does not recognize the failure of that action. This level of interpretability is not perfect for every failure case, but does explain many problems pointing the way for how to improve the model and its training regime.

understand which components caused a failure; see fig. 7 for example attention maps for failed commands and the level of explanation possible along with its limitations. In many cases, this provides both reassurances that errors will be pinpointed to the responsible part of the model and confidence in the chosen model. This level of transparency is unusual for end-to-end models in robotics.

In the future, we plan to extend the language learning capacities of the approach and explore ways to carry out more complex tasks. Thus far we have used an existing off-the-shelf parser, but learning to interpret the sentences while learning to execute commands can be extremely useful - for example it can help disambiguate domain-specific language. In the future we intend to integrate with such work [24, 25, 26] to acquire the structure of language, not just the meanings of words and sentences. The tasks carried out here are concrete and clear, rather than the abstract tasks, e.g., set up this meeting room, that robots should ultimately strive for. We plan to explore ways to break down tasks into components and keep track of the temporal relations between components in the future. 


\section{REFERENCES}

[1] V. Blukis, D. Misra, R. A. Knepper, and Y. Artzi, "Mapping navigation instructions to continuous control actions with position visitation prediction," in Proceedings of the Conference on Robot Learning, 2018.

[2] D. K. Misra, A. Bennett, V. Blukis, E. Niklasson, M. Shatkhin, and Y. Artzi, "Mapping instructions to actions in 3d environments with visual goal prediction," in Proceedings of the 2018 Conference on Empirical Methods in Natural Language Processing, 2018.

[3] P. Shah, M. Fiser, A. Faust, J. C. Kew, and D. Hakkani-Tur, "FollowNet: Robot navigation by following natural language directions with deep reinforcement learning," arXiv preprint arXiv:1805.06150, 2018.

[4] D. Hsu, J.-C. Latombe, and R. Motwani, "Path planning in expansive configuration spaces," in ICRA, 1997.

[5] S. Karaman and E. Frazzoli, "Sampling-based algorithms for optimal motion planning," The International Journal of Robotics Research, vol. 30, no. 7, pp. 846-894, 2011.

[6] B. Chen, B. Dai, and L. Song, "Learning to plan via neural explorationexploitation trees," arXiv preprint arXiv:1903.00070, 2019.

[7] Y.-L. Kuo, A. Barbu, and B. Katz, "Deep sequential models for sampling-based planning," in 2018 IEEE/RSJ International Conference on Intelligent Robots and Systems (IROS). IEEE, 2018, pp. 6490-6497.

[8] L. Lee, E. Parisotto, D. S. Chaplot, E. Xing, and R. Salakhutdinov, "Gated path planning networks," in Proceedings of the 35th International Conference on Machine Learning (ICML 2018), 2018.

[9] S. Tellex, T. Kollar, S. Dickerson, M. R. Walter, A. G. Banerjee, S. Teller, and N. Roy, "Understanding natural language commands for robotic navigation and mobile manipulation," in Twenty-Fifth AAAI Conference on Artificial Intelligence, 2011.

[10] R. Paul, A. Barbu, S. Felshin, B. Katz, and N. Roy, "Temporal grounding graphs for language understanding with accrued visuallinguistic context," in Proceedings of the 26th International Joint Conference on Artificial Intelligence. AAAI Press, 2017, pp. 45064514.

[11] S. M. LaValle, "Rapidly-exploring random trees: A new tool for path planning," 1998.

[12] C. Watkins, "Learning form delayed rewards," Ph. D. thesis, King's College, University of Cambridge, 1989.

[13] S. Bird, "NLTK: the natural language toolkit," in Proceedings of the COLING/ACL on Interactive presentation sessions. Association for Computational Linguistics, 2006, pp. 69-72.

[14] M. Fox and D. Long, "PDDL2. 1: An extension to PDDL for expressing temporal planning domains," Journal of artificial intelligence research, vol. 20, pp. 61-124, 2003.

[15] L. P. Kaelbling and T. Lozano-Pérez, "Integrated task and motion planning in belief space," The International Journal of Robotics Research, vol. 32, no. 9-10, pp. 1194-1227, 2013.

[16] C. R. Garrett, T. Lozano-Perez, and L. P. Kaelbling, "Ffrob: Leveraging symbolic planning for efficient task and motion planning," The International Journal of Robotics Research, vol. 37, no. 1, pp. 104-136, 2018.

[17] N. T. Dantam, S. Chaudhuri, and L. E. Kavraki, "The task-motion kit: An open source, general-purpose task and motion-planning framework," IEEE Robotics \& Automation Magazine, vol. 25, no. 3, pp. 61-70, 2018.

[18] M. Eppe, P. D. Nguyen, and S. Wermter, "From semantics to execution: Integrating action planning with reinforcement learning for robotic tool use," arXiv preprint arXiv:1905.09683, 2019.

[19] C. Paxton, Y. Bisk, J. Thomason, A. Byravan, and D. Fox, "Prospection: Interpretable plans from language by predicting the future," in 2019 International Conference on Robotics and Automation (ICRA), 2019, pp. 6942-6948.

[20] Y. Bisk, D. Yuret, and D. Marcu, "Natural language communication with robots," in Proceedings of the 2016 Conference of the North American Chapter of the Association for Computational Linguistics: Human Language Technologies, 2016, pp. 751-761.

[21] J. Fu, A. Korattikara, S. Levine, and S. Guadarrama, "From language to goals: Inverse reinforcement learning for vision-based instruction following," ICLR, 2019.

[22] G. A. Miller, "WordNet: a lexical database for english," Communications of the ACM, vol. 38, no. 11, pp. 39-41, 1995.

[23] T. Pedersen, S. Patwardhan, and J. Michelizzi, "Wordnet:: Similarity: measuring the relatedness of concepts," in Demonstration papers at HLT-NAACL 2004. Association for Computational Linguistics, 2004, pp. 38-41.
[24] Y. Artzi and L. Zettlemoyer, "Weakly supervised learning of semantic parsers for mapping instructions to actions," Transactions of the Association for Computational Linguistics, vol. 1, pp. 49-62, 2013.

[25] E. C. Williams, N. Gopalan, M. Rhee, and S. Tellex, "Learning to parse natural language to grounded reward functions with weak supervision," in 2018 IEEE International Conference on Robotics and Automation (ICRA). IEEE, 2018, pp. 1-7.

[26] C. Ross, A. Barbu, Y. Berzak, B. Myanganbayar, and B. Katz, "Grounding language acquisition by training semantic parsers using captioned videos," in Proceedings of the 2018 Conference on Empirical Methods in Natural Language Processing, 2018, pp. 2647-2656. 